\title{
Fock Parafermions and Self-Dual Representations of the Braid Group
}

\author{
Emilio Cobanera ${ }^{a, 1}$ and Gerardo Ortiz ${ }^{b}$ \\ ${ }^{a}$ Instituut-Lorentz, Universiteit Leiden, P.O. Box 9506, 2300 RA Leiden, The \\ Netherlands, \\ ${ }^{b}$ Department of Physics, Indiana University, Bloomington, IM 47405, USA.
}

\begin{abstract}
We introduce and describe in second quantization a family of particle species with $p=2,3, \ldots$ exclusion and $\theta=2 \pi / p$ exchange statistics. We call these anyons Fock parafermions, because they are the particles naturally associated to the parafermionic zero-energy modes, potentially realizable in mesoscopic arrays of fractional topological insulators. Their second-quantization description entails the concept of Fock algebra, i.e., a Fock space endowed with a statistical multiplication that captures and logically correlates these anyons' exclusion and exchange statistics. As a consequence normal-ordering continues to be a well-defined operation. Because of its relevance to topological quantum information processing, we also derive families of self-dual representations of the braid group for any $p$, with the Gaussian representation being a special case. The self-dual representations can be realized in terms of local quadratic combinations of either parafermions or Fock parafermions, an important requisite for physical implementation of quantum logic gates.
\end{abstract}

Keywords: anyons in second quantization, Fock space realization of parafermions, dualities, unitary representations of the braid group, Weyl and Generalized Clifford Algebras, noncommutative symplectic geometry

\section{Introduction: The problem of anyons in second quantization}

During the last decade quantum information science has evolved into a mature discipline, with challenging theoretical developments actually result-

\footnotetext{
${ }^{1}$ Corresponding author: cobanera@lorentz.leidenuniv.nl
} 
ing in intense experimental work. Large scale quantum information processing devices may not become readily available anytime soon, but when they do become a reality, they will offer enormous advantages to scientists and engineers. The challenge, or hope rather, that constructing the inner gears of physical reality in the right way may foster quantum information processing is so great that it is forcing us to reconsider fundamental principles. Consider the fundamental aspects of quantum mechanics in two space dimensions. Topological quantum computation exploits braiding at the hardware level to minimize the environment's decohering effects [1], thus constituting an example of utilizing fundamental physical principles as engineering assets.

In this paper we are concerned with the Fock space representation and braiding of anyons, which are indistinguishable particles with unconventional statistics. We call the second quantized description of a particular type of anyon a Fock parafermion. Fock parafermions are tied to localized excitations of clock models [2, 3], edge modes of quantum Hall liquids [4], and fractional topological insulators in general. In this paper we introduce the concept of self-dual representation of the braid group [5] and obtain an extended family of examples. Before describing the specific contents and main results of this paper, let us briefly review the concept of fractional exchange statistics as developed in the framework of first quantization.

The non-relativistic (first quantized) description of $N$ indistinguishable particles, each constrained to a $d$-dimensional coordinate space $\mathbb{R}^{d}$, relies on the analysis of the topological properties of the system's classical physical configuration space $\mathcal{C}_{N}(d)[6,7]$. The latter is defined as a modification of the usual Cartesian product $\mathbb{R}^{d N}$ for distinguishable particles. This modification entails removing the points where two or more particles coincide, and then identifying points that are equal up to a permutation of single-particle indices. Hence each physical configuration of the $N$ indistinguishable particles corresponds to a unique point in $\mathcal{C}_{N}(d)$. The topological properties of $\mathcal{C}_{N}(d)$ are characterized by the homotopy equivalences among physical trajectories that effectively exchange two particle indices. The particles' statistical exchange properties should be independent of their trajectories; two trajectories that can be continuously deformed to each other are equivalent. As it turns out, the first homotopy group of the configuration space is the permutation group of $N$ objects, $S_{N}$, whenever $d \geq 3$, and it is the braid group $B_{N}$ of $N$ strands for $d=2[7]$. This implies that in higher space dimensions particles may be either fermions or bosons while in $d=2$, there are possibilities for more exotic particles, such as anyons, which show fractional statistics. 
It is important to emphasize that this way of characterizing particle statistics is kinematical, meaning that it does not invoke any particular Hamiltonian. Indeed, one can define exchange statistics as a phenomenon of parallel transport (without Hamiltonians) and the statistical phase as the holomony associated with the geometric evolution leading to the exchange path, i.e., a statistical evolution. This is analogous to the definition of Berry phases and its generalizations [8], which are holonomies, associated with adiabatic parallel transport over a generic parameter space. Based on the character of the physical state, which must belong to a Hilbert space, one can realize statistical evolutions which may be Abelian or non-Abelian, depending on the dimension of the unitary matrix representing the holonomy. Thus, one may speak of non-Abelian exchange statistics whenever that matrix is at least two-dimensional while the simple scalar case will be reserved for Abelian statistics.

Only multi-component states allow for non-Abelian statistical evolutions. In practice one can simulate a multi-component state by choosing a subspace degenerate in energy or some other conserved quantity, such as the ground state of a Hamiltonian. Then one can perform an adiabatic evolution to realize the desired non-Abelian exchange. The implementation of non-Abelian statistical phases is dynamical and subject to errors, including departures from adiabaticity. This particular type of error can be systematically accounted for by using an adiabatic perturbation theory [9]. Holonomic quantum computation [1] takes this observation and extends its scope by exploiting adiabatic transport in arbitrary non-Abelian unitary vector bundles to achieve quantum logic gates. Topological quantum computation [1] is a particular instance of holonomic computation where the transported subspace of degenerate states has special physical properties. Typically, it is the manifold of ground states for topological quantum matter that are said to be topologically protected or robust against local perturbations. The aim of topological quantum computation is to exploit this fact at the hardware level for fault tolerant quantum information processing.

Topological quantum matter represents many-body systems whose degenerate ground states cannot be distinguished by local measurements in the bulk [10]. They typically support localized modes or excitations that may be carefully manipulated to effectively exhibit anyonic exchange statistics. For example $[11,12,13]$, it is common to hear that Majorana modes are nonAbelian anyons, because they support a non-Abelian, unitary representation of the braid group. However, Majoranas are not particles. By particle, we 
mean the usual quantum field theory notion, which requires a Fock space and thus, second quantization; that is, one needs a way of counting them. That said, by generalizing the concept of particle statistics to localized excitations of topologically ordered vacua, we acquire a new source of robustness. These localized excitations support natural representations of the braid group and are optimal candidates for the realization of the elusive topological quantum computer.

In this paper, we study a generalization of Majorana modes called Weyl parafermions [14]. Parafermions are effective degrees of freedom or modes associated to clock models $[17,18]$ that, as we will show, support a variety of non-Abelian representations of the braid group (including the Majorana representation as a special case). Moreover, they are naturally associated to particle degrees of freedom that we call Fock parafermions. Fock parafermions are indistinguishable particles displaying unconventionally correlated $p$-exclusion and $\frac{2 \pi}{p}$-exchange statistics, where $p$ is an integer larger than one. Most importantly, we introduce self-dual representations of the braid group for all $p$ which can be realized in terms of quadratic combinations of either Weyl or Fock parafermions, a crucial asset for physical implementation.

The outline of the paper follows. In Section 2 we present the Weyl algebra with generators $U$ and $V$ originally introduced to study finite-dimensional quantum mechanics. There we show a fundamental canonical decomposition of the algebra in terms of Weyl algebras with smaller periods. This technical result is used to analyze the self-dual representations of the braid group developed in later sections. It turns out that the Weyl algebra is behind the realization of Weyl parafermions, or simply parafermions, with Majoranas being identified with their lower dimensional representation $p=2$. This is the topic of Section 3, where we derive parafermions as the product of order and disorder variables associated with self-dual quantum clock models [3]. Next we present the self-dual representations of the braid group in Section 4 , which is one of the main results of the paper, and we show how to realize these representations in terms of parafermions. We also show that the Gaussian representation of Jones $[19,20]$ is a particular example of a selfdual representation. Our next main result is unveiled in Section 5, where we show how to construct a Fock space for the Fock parafermions that reduce to canonical fermions in the case $p=2$. The key technical development is the introduction of the concept of Fock algebra with a statistical multiplication. The advantage of this approach is that it allows us to derive the unconven- 
tional algebra of creation and destruction operators of Fock parafermions. It is possible that this approach may be generalized to obtain Fock spaces for anyon-like particles with more complicated statistical properties. Majoranas are mathematically realized as combinations of fermionic creation and annihilation operators. Fock parafermions possess an unconventional algebra of creation and annihilation operators which allows us to generalize this connection to all Weyl parafermions, as shown in Section 6. Finally, Appendix A describes a Jordan-Wigner-like relation between Fock parafermions and a new type of particle that we call Weyl hard-core bosons, which have a Fock space representation with $p$-exclusion and bosonic statistics.

\section{Finite-dimensional quantum mechanics: Weyl algebra}

Heisenberg's canonical commutation relations for position, $X_{i}$, and momentum, $P_{i}$, Hermitian operators

$$
\left[X_{i}, P_{j}\right]=\mathrm{i} \hbar \delta_{i, j} \mathbb{1}, \quad i, j=1, \ldots, M,
$$

cannot be satisfied by finite-dimensional matrix representations [21]. This is unfortunate because it is natural to seek insight into quantum mechanics by disentangling conceptual issues from the mathematical complications associated to infinite-dimensional state spaces. To realize a finite-dimensional framework for quantum mechanics, Hermann Weyl [22, 23] proposed a different starting point: the algebra $W(M)$ generated by translations in position and momentum spaces,

$$
V_{i, \mathrm{x}} \equiv e^{\mathrm{i} \times P_{i} / \hbar}, \quad U_{j, \mathrm{p}} \equiv e^{\mathrm{ip} X_{j} / \hbar}, \quad i, j=1, \ldots, M .
$$

These unitary generators commute for $i \neq j$, and otherwise satisfy

$$
V_{i, \mathrm{x}} U_{i, \mathrm{p}}=e^{\mathrm{ixp} / \hbar} U_{i, \mathrm{p}} V_{i, \mathrm{x}}
$$

The Weyl algebra $W(M)$ does admit a natural finite-dimensional truncation. Let us introduce a lattice (fundamental) spacing $\delta$, and discretize $\mathrm{x}, \mathrm{p}$ as

$$
\mathrm{x}_{m}=m \delta, \quad \mathrm{p}_{n}=\frac{2 \pi \hbar n}{p \delta}, \quad m, n \in \mathbb{Z},
$$

with $p=2,3, \ldots$ a fixed positive integer. Then, we can define

$$
V_{i} \equiv e^{\mathrm{i} \delta P_{i} / \hbar}, \quad U_{j} \equiv e^{\mathrm{i} 2 \pi X_{j} / p \delta},
$$


so that $V_{i, \times_{n}}=V_{i}^{n}, U_{j, \mathrm{p}_{m}}=U_{j}^{m}$, and

$$
V_{i} U_{i}=\omega U_{i} V_{i}, \quad \omega \equiv e^{\mathrm{i} 2 \pi / p}, \quad \bar{\omega}=e^{-\mathrm{i} 2 \pi / p} .
$$

It was Weyl who noticed [22] that the relations of Eq. (6) do admit a finite dimensional, unitary representation $W_{p}(M)$ in a Hilbert space (defined over the field of complex numbers C) of dimension $p^{M}$. In particular, the unitary $p \times p$ matrices

$$
V=\left(\begin{array}{ccccc}
0 & 1 & 0 & \cdots & 0 \\
0 & 0 & 1 & \cdots & 0 \\
0 & 0 & 0 & \cdots & 0 \\
\vdots & \vdots & \vdots & & \vdots \\
0 & 0 & 0 & \cdots & 1 \\
1 & 0 & 0 & \cdots & 0
\end{array}\right), \quad U=\left(\begin{array}{ccccc}
1 & 0 & 0 & \cdots & 0 \\
0 & \omega & 0 & \cdots & 0 \\
0 & 0 & \omega^{2} & \cdots & 0 \\
\vdots & \vdots & \vdots & & \vdots \\
0 & 0 & 0 & \cdots & 0 \\
0 & 0 & 0 & \cdots & \omega^{p-1}
\end{array}\right) .
$$

satisfy $V U=\omega U V$, and have finite period $p\left(V^{p}=\mathbb{1}=U^{p}\right)$. Hence the Weyl generators

$$
\begin{aligned}
V_{i} & \equiv \mathbb{1} \otimes \cdots \otimes V \otimes \cdots \otimes \mathbb{1}, \quad(i \text { th position }), \quad i=1, \ldots, M, \\
U_{j} & \equiv \mathbb{1} \otimes \cdots \otimes U \otimes \cdots \otimes \mathbb{1}, \quad(j \text { th position }), \quad j=1, \ldots, M,
\end{aligned}
$$

afford the desired finite-dimensional, unitary representation of Eq. (6). For $p=2, \omega=-1, V=\sigma^{x}$, and $U=\sigma^{z}$ (where $\sigma^{x, z}$ are Pauli matrices). It is interesting to notice that while Planck's constant explicitly appears in $W(M)$, it does not appear in $W_{p}(M)$, and so there is no obvious notion of classical, $\hbar \rightarrow 0$, limit for this form of discrete quantum mechanics.

It is well known that the three Pauli matrices $\sigma^{x}, \sigma^{z}, \sigma^{y}=\mathrm{i} \sigma^{x} \sigma^{z}$ close a Lie algebra. There is an interesting generalization of this fact to arbitrary $p$. Let $\mathbf{m}=\left(m_{1}, m_{2}\right)$ with $m_{1,2}=0, \ldots, p-1$, non-negative integers. Define the unitary Schwinger basis

$$
J_{\mathbf{m}} \equiv \bar{\omega}^{m_{1} m_{2} / 2} V^{m_{1}} U^{m_{2}} .
$$

The set $\left\{J_{\mathbf{m}}\right\}$ realizes an orthogonal (with respect to the Hermitian inner product defined by the trace) basis of the full matrix algebra $M_{p}(\mathbb{C})$ of $p \times p$ complex matrices. Moreover, the Schwinger basis is closed under the bracket operation,

$$
\left[J_{\mathbf{m}}, J_{\mathbf{n}}\right]=2 \mathbf{i} \sin \left(\frac{\pi}{p}(\mathbf{m} \times \mathbf{n})\right) J_{\mathbf{m}+\mathbf{n}} .
$$


Throughout this paper, addition and multiplication of integers are understood to be modulo $p$ operations unless stated otherwise. Discarding the identity $J_{(0,0)}=1$, we recognize this to be the Lie algebra of $p$-dimensional complex, traceless matrices $s u(p-1)[24]$.

The Weyl algebra $W_{p}(M)$ finds one of its most important applications in the design of model Hamiltonians with unconventional phase transitions $[2,3]$, non-commutative symplectic geometry [25, 26], measurement-assisted topological quantum computation [4], and the study of interacting zeroenergy edge modes [29]. Historically, 't Hooft's work on confinement in QCD was instrumental to popularizing Weyl's algebra in the late 1970s. In Ref. [30], he proposed a generalized order parameter for $S U(N)$ gauge theories satisfying the algebra of the Weyl generators. A flurry of activity followed, centered on studying quantum many-body systems with Weyl generators as elementary degrees of freedom. Popular examples are the vector Potts, or $p$-clock, model for the BKT phase transition [2], $\mathbb{Z}_{p}$ gauge theories for confinement transitions [31], and, more recently, topologically ordered $\mathbb{Z}_{p}$ toric code models [32].

In closing this section on Weyl algebras, we want to show that the Weyl algebra $W_{p}$ admits a canonical decomposition in terms of Weyl algebras $W_{p_{j}}$ with periods $p_{j}$,

$$
W_{p_{1}} \otimes \cdots \otimes W_{p_{t}} \cong W_{p}
$$

dictated by the decomposition of $p$ into relative prime factors,

$$
p=\prod_{j=1}^{t} p_{j}, \quad p_{j}=q_{j}^{m_{j}}, \quad q_{j} \text { distinct prime numbers, } j=1, \ldots, t,
$$

with $m_{j}$ and $t$ integers. The isomorphic mapping $\cong$ will be very useful for establishing some basic properties of the self-dual representations of the braid group in Section 4.

Assume $p=p_{1} p_{2}$, with $p_{1}$ and $p_{2}$ relative primes. Then the mapping

$$
U_{\left(p_{1}\right)}^{m} \otimes U_{\left(p_{2}\right)}^{m} \cong U_{(p)}^{m}, \quad V_{\left(p_{1}\right)}^{m} \otimes V_{\left(p_{2}\right)}^{m} \cong V_{(p)}^{\left(p_{1}+p_{2}\right) m}, \quad m=0, \ldots, p-1,
$$

induces the algebra isomorphism

$$
W_{p_{1}} \otimes W_{p_{2}} \cong W_{p}
$$

(Here we write $U_{\left(p_{j}\right)}, V_{\left(p_{j}\right)}$ for the Weyl generators of $W_{p_{j}}$ instead of $U, V$, and $\omega_{(p)}$ instead of $\omega$. For example, $\omega_{(2)}=-1, U_{(2)}=\sigma^{z}$ and $V_{(2)}=\sigma^{x}$.) To 
see that $\cong$ is an algebra isomorphism we need to combine two observations. First, the two relations

$$
\begin{aligned}
\left(V_{\left(p_{1}\right)}^{m} \otimes V_{\left(p_{2}\right)}^{m}\right)\left(U_{\left(p_{1}\right)}^{n} \otimes U_{\left(p_{2}\right)}^{n}\right) & =\omega_{\left(p_{1}\right)}^{m n} \omega_{\left(p_{2}\right)}^{m n}\left(U_{\left(p_{1}\right)}^{n} \otimes U_{\left(p_{2}\right)}^{n}\right)\left(V_{\left(p_{1}\right)}^{m} \otimes V_{\left(p_{2}\right)}^{m}\right) \\
V_{(p)}^{\left(p_{1}+p_{2}\right) m} U_{(p)}^{n} & =\omega_{p}^{\left(p_{1}+p_{2}\right) m n} U_{(p)}^{n} V_{(p)}^{\left(p_{1}+p_{2}\right) m}
\end{aligned}
$$

are satisfied because

$$
\omega_{\left(p_{1}\right)}^{m n} \omega_{\left(p_{2}\right)}^{m n}=\omega_{(p)}^{\left(p_{1}+p_{2}\right) m n} .
$$

Second, the mapping

$$
m \mapsto\left(p_{1}+p_{2}\right) m(\bmod p), \quad m=0, \ldots, p-1,
$$

is one-to-one and onto, but only if $p_{1}, p_{2}$ are relative primes. The isomorphism fails to be one-to-one if one does not enforce this latter condition. For example, blindly applied to the case $p_{1}=2, p_{2}=2, p=4$, Eq. (14) obtains

$$
\sigma^{x} \otimes \sigma^{x} \cong V_{(4)}^{4}=\mathbb{1}
$$

mapping an element that is not the identity to the identity. Since by definition the factors $p_{j}$ in Eq. (13) are relative primes, repeated application of Eq. (14) establishes the decomposition of Eq. (12).

Consider as an example the decomposition $W_{2} \otimes W_{3} \cong W_{6}$ :

$$
\begin{array}{rlrl}
\sigma^{z} \otimes U_{(3)} & \cong U_{(6)}, & \sigma^{x} \otimes V_{(3)} \cong V_{(6)}^{5}, \\
\mathbb{1}_{(2)} \otimes U_{(3)}^{2} & \cong U_{(6)}^{2}, & \mathbb{1}_{(2)} \otimes V_{(3)}^{2} \cong V_{(6)}^{4}, \\
\sigma^{z} \otimes \mathbb{1}_{(3)} \cong U_{(6)}^{3}, & \sigma^{x} \otimes \mathbb{1}_{(3)} \cong V_{(6)}^{3}, \\
\mathbb{1}_{(2)} \otimes U_{(3)} \cong U_{(6)}^{4}, & \mathbb{1}_{(2)} \otimes V_{(3)} \cong V_{(6)}^{2}, \\
\sigma^{z} \otimes U_{(3)}^{2} & \cong U_{(6)}^{5}, & \sigma^{x} \otimes V_{(3)}^{2} \cong V_{(6)} .
\end{array}
$$

The reasoning leading to $\cong$ can be adapted to obtain canonical embeddings $W_{q} \hookrightarrow W_{p}$ of lower into higher dimensional Weyl algebras. If $p / q$ is an integer and $p / q$ and $q$ are relative primes, then the mapping

$$
U_{(q)}^{m p / q} \hookrightarrow U_{(p)}^{m p / q}, \quad V_{(q)}^{m p / q} \hookrightarrow V_{(p)}^{m p^{2} / q^{2}}, \quad m=0, \ldots, q-1,
$$

defines a one-to-one homomorphism. We can read off our example in the previous paragraph the embeddings $W_{2} \hookrightarrow W_{6}$ and $W_{3} \hookrightarrow W_{6}$,

$$
\begin{array}{rlll}
\sigma^{z} & \hookrightarrow U_{(6)}^{3}, & \sigma^{x} \hookrightarrow V_{(6)}^{3}, \\
U_{(3)} & \hookrightarrow U_{(6)}^{4}, & V_{(3)} \hookrightarrow V_{(6)}^{2}, \\
U_{(3)}^{2} & \hookrightarrow U_{(6)}^{2}, & V_{(3)}^{2} & \hookrightarrow V_{(6)}^{4} .
\end{array}
$$


Similarly to the isomorphism $\cong$, the embeddings $\hookrightarrow$ will be very useful to understand some properties of our self-dual representations of the braid group in Section 4.

\section{Weyl parafermions}

In this section we want to illustrate the natural relation between Majorana fermions and the finite-dimensional, $p=2$, representation of the Weyl algebra defined in previous section. This fact allows a generalization of the Majorana concept to higher-dimensional representations, $p>2$, in terms of quantum degrees of freedom called Weyl parafermions or, simply, parafermions.

The operators

$$
\Gamma_{i} \equiv V_{i}\left(\prod_{j=1}^{i-1} U_{j}\right), \quad \Delta_{i} \equiv V_{i} U_{i}\left(\prod_{j=1}^{i-1} U_{j}\right)=\Gamma_{i} U_{i}
$$

define an alternative set of generators of the Weyl algebra $W_{p}(M)$, as follows from the inverse relations

$$
U_{i}=\Gamma_{i}^{\dagger} \Delta_{i}, \quad V_{i}=\Gamma_{i}\left(\prod_{j=1}^{i-1} \Delta_{j}^{\dagger} \Gamma_{j}\right)
$$

The new generators satisfy

$$
\begin{gathered}
\Gamma_{i} \Gamma_{j}=\omega \Gamma_{j} \Gamma_{i}, \quad \Delta_{i} \Delta_{j}=\omega \Delta_{j} \Delta_{i}, \quad \text { for } \quad i<j \\
\Gamma_{i} \Delta_{j}=\omega \Delta_{j} \Gamma_{i}, \quad \text { for } \quad i \leq j
\end{gathered}
$$

and

$$
\Gamma_{i}^{p}=\mathbb{1}=\Delta_{i}^{p}, \quad \Gamma_{i}^{p-1}=\Gamma_{i}^{\dagger}, \quad \Delta_{i}^{p-1}=\Delta_{i}^{\dagger} .
$$

Regarded as quantum degrees of freedom, the $\Gamma_{i}, \Delta_{i}$ are collectively called parafermions $[18,3]$. We will come back to the physical meaning and the context in which parafermionic excitations emerge later in this section.

In the mathematical literature, the algebra realized by parafermions [33] is known as a generalized Clifford algebra [34, 35, 36]. This is in part because parafermions generate the standard Clifford algebra for the special case $p=2$. Let

$$
a_{i} \equiv \Gamma_{i}, \quad \mathrm{i} b_{i} \equiv-\Delta_{i}, \quad \text { for } p=2
$$


Then, from Eq. (26),

$$
a_{i}=\sigma_{i}^{x}\left(\prod_{j=1}^{i-1} \sigma_{j}^{z}\right), \quad b_{i}=\sigma_{i}^{y}\left(\prod_{j=1}^{i-1} \sigma_{j}^{z}\right),
$$

so that $a_{i}^{\dagger}=a_{i}, b_{i}^{\dagger}=b_{i}$, and Eqs. (28), (29), and (30) can be recast in the form most familiar for a Clifford algebra

$$
\left\{a_{i}, a_{j}\right\}=2 \delta_{i, j}, \quad\left\{b_{i}, b_{j}\right\}=2 \delta_{i, j}, \quad\left\{a_{i}, b_{j}\right\}=0 .
$$

Regarded as quantum degrees of freedom, the operators $a_{i}, b_{i}$ are known as Majorana fermions, or just Majoranas for short, in recent condensed matter and quantum computation literature [13, 37, 38, 39, 11].

There is one key piece of information that sets a drastic divide between $p=2$ and $p \geq 3$. Following the relations

$$
a_{i}=C_{i}+C_{i}^{\dagger}, \quad \mathrm{i} b_{i}=C_{i}-C_{i}^{\dagger},
$$

Majoranas can be combined to yield creation $C_{i}^{\dagger}$ and annihilation $C_{i}$ operators of fermionic particles,

$$
\left\{C_{i}, C_{j}^{\dagger}\right\}=\delta_{i, j}, \quad\left\{C_{i}, C_{j}\right\}=0=\left\{C_{i}^{\dagger}, C_{j}^{\dagger}\right\}, \quad i, j=1, \ldots, M .
$$

It is textbook knowledge that this algebra, i.e., the canonical anti-commutation relations of Eq. (35), determines completely the Fock space for indistinguishable fermions with $M$ available orbitals [40]. So even though Majorana fermions are not particles (not even in the effective sense of Landau quasiparticles), they are tightly linked to fermionic particles and the associated fermionic Fock space. In Section 5 we will show that there exists a Fock space representation of parafermions for all $p$. The associated indistinguishable particles satisfy unconventional exclusion and exchange statistics. We call these particles Fock parafermions.

Parafermions can be defined in more than one spatial dimension, provided one establishes an order for the set of indices labelling the Weyl generators. Suppose for concreteness that Weyl generators $V_{\boldsymbol{r}}, U_{\boldsymbol{r}}$ are placed at each site $\boldsymbol{r}$ of a hypercubic lattice of finite extent $M$ in each of its $d$ spatial directions, that is, $\boldsymbol{r}=\left(m^{1}, \ldots, m^{d}\right)$ with $m^{\mu}=1, \ldots, M, \mu=1,2, \cdots, d$. Then the sites $\boldsymbol{r}$ can be ordered lexicographically. For example, in $d=2$ dimensions, a possible order is

$$
(1,1)<(1,2)<\cdots<(1, M)<(2,1)<\cdots<(M, M-1)<(M, M) .
$$


Once an order is established, parafermions can be defined as

$$
\Gamma_{r}=V_{r} \prod_{x<r} U_{x}, \quad \Delta_{r}=V_{r} U_{r} \prod_{x<r} U_{x},
$$

in any number of spatial dimensions, and still satisfy the generalized Clifford algebra of Eqs. (28), (29) with respect to that chosen order. For the special case $p=2$, these considerations together with Eq. (34) lead directly to the Jordan-Wigner transformation for Majorana fermions in more than one space dimension [16].

Parafermions have a specially transparent physical interpretation in one dimension where they arise in the study of self-dual clock models $[2,3]$. The simplest instance is the vector Potts [41], or $p$-clock, model $[2,3]$

$$
H_{\mathrm{VP}}\left[h_{i}, J_{i}\right]=-\frac{1}{2}\left[\sum_{i=1}^{M} h_{i} U_{i}+\sum_{i=1}^{M-1} J_{i} V_{i} V_{i+1}^{\dagger}+J_{M} V_{M}\right]+\text { h.c. . }
$$

The boundary term $J_{M}\left(V_{M}+V_{M}^{\dagger}\right)$ enforces boundary conditions chosen to showcase the presence of an exact symmetry, not of the Hamiltonian but rather of the model's bond algebra of interactions $[42,3]$. Let

$$
r(i)=M+1-i .
$$

denote the reflection through the system's midpoint. Then the mapping

$$
\begin{array}{rlll}
U_{1} & \stackrel{\Phi_{\mathrm{d}}}{\longrightarrow} V_{r(1)}^{\dagger}=V_{M}^{\dagger}, & \\
U_{i} & \stackrel{\Phi_{\mathrm{d}}}{\longrightarrow} V_{r(i)}^{\dagger} V_{r(i)+1}, & i=2, \ldots, M, \\
V_{i} V_{i+1}^{\dagger} & \stackrel{\Phi_{\mathrm{d}}}{\longrightarrow} & U_{r(i)} & i=1, \ldots, M-1, \\
V_{M} & \stackrel{\Phi_{\mathrm{d}}}{\longrightarrow} & U_{r(M)}=U_{1}, &
\end{array}
$$

local in the model's "interactions" (or more precisely, bonds [3]), induces an algebra isomorphism $\Phi_{\mathrm{d}}$. According to the general theory of dualities developed in Ref. [3], the mapping $\Phi_{\mathrm{d}}$ is unitarily implementable. This means that there exists a unitary transformation $\mathcal{U}_{\mathrm{d}}$ such that

$$
\mathcal{O} \stackrel{\Phi_{\mathrm{d}}}{\longrightarrow} \widehat{\mathcal{O}}, \quad \widehat{\mathcal{O}} \equiv \Phi_{\mathrm{d}}(\mathcal{O})=\mathcal{U}_{\mathrm{d}} \mathcal{O} \mathcal{U}_{\mathrm{d}}^{\dagger}
$$

for any operator $\mathcal{O}$. 
The importance of these observations follow from the effect of the duality mapping on the vector Potts hamiltonian. Since

$$
H_{\mathrm{VP}}\left[h_{i}, J_{i}\right] \stackrel{\Phi_{\mathrm{d}}}{\longrightarrow} H_{\mathrm{VP}}\left[h_{i}^{*}, J_{i}^{*}\right],
$$

with dual couplings

$$
h_{i}^{*} \equiv J_{r(i)}, \quad J_{i}^{*} \equiv h_{r(i)}
$$

it follows that

$$
H_{\mathrm{VP}}\left[h_{i}^{*}, J_{i}^{*}\right]=\mathcal{U}_{\mathrm{d}} H_{\mathrm{VP}}\left[h_{i}, J_{i}\right] \mathcal{U}_{\mathrm{d}}^{\dagger} .
$$

That is, any pair of vector Potts Hamiltonians associated to points $\left\{h_{i}, J_{i}\right\}$ and $\left\{h_{i}^{*}, J_{i}^{*}\right\}$ in coupling space are isospectral. In more physical terms, the vector Potts model is self-dual.

Now that we understand the model's self-duality in detail we can restore conventional open boundary conditions by setting $J_{M}=0$. By restoring conventional boundary conditions we also recover the the model's global $\mathbb{Z}_{p}$ symmetry,

$$
Q_{p}=\prod_{i=1}^{M} U_{i}, \quad\left[H_{\mathrm{VP}}\left[J_{M}=0\right], Q_{p}\right]=0 .
$$

The dual Hamiltonian however

$$
H_{\mathrm{VP}}\left[h_{1}^{*}=0\right]=\mathcal{U}_{\mathrm{d}} H_{\mathrm{VP}}\left[J_{M}=0\right] \mathcal{U}_{\mathrm{d}}^{\dagger}
$$

is not globally symmetric, but rather has a boundary symmetry as can be checked explicitly. From the point of view of the duality transformation this follows because

$$
Q_{p} \stackrel{\Phi_{\mathrm{d}}}{\longrightarrow} V_{1}^{\dagger}
$$

and so $V_{1}^{\dagger}$ must commute with $H_{\mathrm{VP}}\left[h_{1}^{*}=0\right]$. This localized symmetry is an elementary example of a holographic symmetry [43], that is, a boundary symmetry dual to a bulk symmetry. As explained in Ref. [43], holographic symmetries are valuable stepping stones in the search for interacting zero-energy edge modes and generalized order parameters for topologically-ordered quantum phases of matter.

Since the duality mapping $\Phi_{\mathrm{d}}$ is an isomorphism, the dual variables

$$
\widehat{V}_{i} \equiv \Phi_{\mathrm{d}}\left(V_{i}\right), \quad \widehat{U}_{i} \equiv \Phi_{\mathrm{d}}\left(U_{i}\right)
$$

satisfy all the relations expected of a set of Weyl generators. Moreover, in view of this paper's notation for the vector Potss model [41], the quantum 
variable $V_{i}$ is associated to the model's order parameter. Hence it is conventional to call the $V_{i}$ order variables, and the

$$
\widehat{V}_{i}=\Phi_{\mathrm{d}}\left(\left(V_{i} V_{i+1}^{\dagger}\right) \ldots\left(V_{M-1} V_{M}^{\dagger}\right) V_{M}\right)=U_{r(i)} \ldots U_{1}
$$

disorder variables (see [3] for the relation of disorder variables to disorder parameters). Recalling the definition of parafermions, Eq. (26), we see that $\left(\widehat{V}_{M+1} \equiv \mathbb{1}\right)$

$$
\Gamma_{i}=V_{i} \widehat{V}_{r(i)+1}, \quad \Delta_{i}=V_{i} \widehat{V}_{r(i)}, \quad i=1, \ldots, M .
$$

That is, in one-dimension, parafermions arise as the product of an order with a disorder variable. This remarkable connection between self-duality and parafermions was first noticed in the early days of the operator product expansion, in the context of classical statistical mechanics [44, 17], and later extended to quantum clock models in Ref. [3].

In closing, let us notice that the $\mathbb{Z}_{p}$-symmetric $\left(J_{M}=0\right)$ vector Potts model can be rewritten as a local (quadratic) Hamiltonian of parafermions,

$$
H_{\mathrm{VP}}=-\frac{1}{2}\left[\sum_{i=1}^{M} h_{i} \Gamma_{i}^{\dagger} \Delta_{i}+\sum_{i=1}^{M-1} J_{i} \Delta_{i} \Gamma_{i+1}^{\dagger}\right]+\text { h.c. . }
$$

\section{Weyl algebras, parafermions, and self-dual representations of the braid group}

Majoranas may emerge as effective excitations or localized zero-energy modes in some topologically-ordered electronic phases of matter, and several current experiments on nanowires are specifically designed to hunt for them [45]. Starting with the key observation that Majoranas realize a twodimensional representation of the braid group [13], protocols have been developed to braid Majoranas and experimentally achieve, with some degree of topological protection, (non-universal) Clifford gates [37, 46, 12].

Since Majorana fermions are but the special $p=2$ instance of parafermions, it is natural to search for platforms and protocols that exploit parafermions with $p>2$ for fault-tolerant quantum information processing $[1,47$, $28,4]$. A necessary first step in this direction is to find parafermionic representations of the braid group. As a general rule, these representations should be as physically motivated as possible to foster experimental realization. Two conditions come immediately to mind: The representations should be unitary 
and local in terms of parafermions. These conditions are motivated by general features of the quantum control model of quantum computation [47]. We will add to this list of generic conditions a third one specific to parafermions: the representations should be self-dual.

As explained in previous sections, the link between parafermions, order, and dual disorder variables offers one of the most compelling physical realizations of parafermions. In the following we will outline a systematic search of parafermionic representations of the braid group, for all values of $p$, that transform in a natural way, Eq. (63), under duality. These are the self-dual representations of the braid group alluded to.

In this paper, by Artin braid group, or simply braid group, we mean the group $B_{L}$ with $L-1$ generators $\sigma_{k}$ and standard "braid relations" [5]

$$
\left.B_{L}=\left\langle\sigma_{1}, \ldots, \sigma_{L-1}\right| \sigma_{k} \sigma_{k+1} \sigma_{k}=\sigma_{k+1} \sigma_{k} \sigma_{k+1}, \sigma_{k} \sigma_{l}=\sigma_{l} \sigma_{k} \text { if }|k-l| \geq 2\right\rangle,
$$

associated to the quantum-mechanical description of $L$ indistinguishable anyons moving in the infinite plane $\mathbb{R}^{2}$. The mapping

$$
\sigma_{k} \mapsto \sigma_{L-k}, \quad k=1, \ldots, L-1,
$$

preserves all the braiding relations and so induces an automorphism of $B_{L}$ that we will have the occasion to use later in this section.

Next we search for unitary representations of the braid group $B_{2 M+1}$ of the form

$$
\begin{array}{rlrl}
\rho_{\mathrm{sd}}^{(p)}\left(\sigma_{2 i-1}\right) & =\frac{1}{\sqrt{p}} \sum_{m=0}^{p-1} \alpha_{m} U_{i}^{\dagger m}, & & i=1, \ldots, M, \\
\rho_{\mathrm{sd}}^{(p)}\left(\sigma_{2 i}\right) & =\frac{1}{\sqrt{p}} \sum_{m=0}^{p-1} \beta_{m}\left(V_{i} V_{i+1}^{\dagger}\right)^{m}, & i=1, \ldots, M-1, \\
\rho_{\mathrm{sd}}^{(p)}\left(\sigma_{2 M}\right) & =\frac{1}{\sqrt{p}} \sum_{m=0}^{p-1} \beta_{m}\left(V_{M}\right)^{m} .
\end{array}
$$

The complex parameters $\alpha_{m}, \beta_{m}, m=0, \ldots, p-1$, are constrained by two requirements: unitarity,

$$
\left(\rho_{\mathrm{sd}}^{(p)}\left(\sigma_{k}\right)\right)^{\dagger}=\rho_{\mathrm{sd}}^{(p)}\left(\sigma_{k}^{-1}\right)
$$

and the braiding relations

$$
\rho_{\text {sd }}^{(p)}\left(\sigma_{k}\right) \rho_{\text {sd }}^{(p)}\left(\sigma_{k+1}\right) \rho_{\text {sd }}^{(p)}\left(\sigma_{k}\right)=\rho_{\text {sd }}^{(p)}\left(\sigma_{k+1}\right) \rho_{\text {sd }}^{(p)}\left(\sigma_{k}\right) \rho_{\text {sd }}^{(p)}\left(\sigma_{k+1}\right) .
$$


Unitarity implies the set of equations (the overbar denotes complex conjugation)

$$
\sum_{m=0}^{p-1} \alpha_{q+m} \bar{\alpha}_{m}=\sum_{m=0}^{p-1} \beta_{q+m} \bar{\beta}_{m}=\left\{\begin{array}{ll}
p & \text { if } q=0 \\
0 & \text { otherwise }
\end{array} .\right.
$$

The braiding relations on the other hand require that

$$
\alpha_{r} \sum_{m+n=q(\bmod p)} \omega^{r m} \beta_{m} \beta_{n}=\beta_{q} \sum_{m+n=r(\bmod p)} \omega^{q m} \alpha_{m} \alpha_{n}, \quad r, q=0, \ldots, p-1 .
$$

We call (the representation associated to) any non-trivial solution of Eqs. (61) and (62) a self-dual representation of the braid group. Self-dual representations are physically distinguished because they transform in a natural fashion under the duality mapping $\mathcal{U}_{\mathrm{d}}$ described in Section 3. By this we mean the following. The duality transformation $\mathcal{U}_{\mathrm{d}}$ can be combined with the automorphism of the braid group Eq. (55) to define out of any given self-dual representation a new representation $\rho^{D}$,

$$
\rho^{D}\left(\sigma_{k}\right) \equiv \mathcal{U}_{\mathrm{d}} \rho_{\mathrm{sd}}^{(p)}\left(\sigma_{r(k)+M}\right) \mathcal{U}_{\mathrm{d}}^{\dagger}
$$

$\left(r(k)\right.$ was defined in Eq. (38)). The point we want to emphasize is that $\rho^{D}$ is again of the self-dual form,

$$
\begin{array}{rlrl}
\rho^{D}\left(\sigma_{2 i-1}\right) & =\frac{1}{\sqrt{p}} \sum_{m=0}^{p-1} \alpha_{m}^{*} U_{i}^{\dagger m}, & i=1, \ldots, M, \\
\rho^{D}\left(\sigma_{2 i}\right) & =\frac{1}{\sqrt{p}} \sum_{m=0}^{p-1} \beta_{m}^{*}\left(V_{i} V_{i+1}^{\dagger}\right)^{m}, & i=1, \ldots, M-1, \\
\rho^{D}\left(\sigma_{2 M}\right) & =\frac{1}{\sqrt{p}} \sum_{m=0}^{p-1} \beta_{m}^{*}\left(V_{M}\right)^{m},
\end{array}
$$

with dual "couplings"

$$
\alpha_{m}^{*}=\beta_{m}, \quad \beta_{m}^{*}=\alpha_{p-m}, \quad m=0, \ldots, p-1 .
$$

A self-dual representation of $B_{2 M}$ can be obtained by dropping $\rho_{\mathrm{sd}}^{(p)}\left(\sigma_{2 M}\right)$. From the point of view of measurement-assisted topological quantum computation, these representations are the most interesting ones because they 
are local and quadratic in terms of parafermions,

$$
\rho_{\mathrm{sd}}^{(p)}\left(\sigma_{2 i-1}\right)=\frac{1}{\sqrt{p}} \sum_{m=0}^{p-1} \alpha_{m}\left(\Delta_{i}^{\dagger} \Gamma_{i}\right)^{m}, \quad \rho_{\mathrm{sd}}^{(p)}\left(\sigma_{2 i}\right)=\frac{1}{\sqrt{p}} \sum_{m=0}^{p-1} \beta_{m}\left(\Delta_{i} \Gamma_{i+1}^{\dagger}\right)^{m}
$$

They are also reducible, since they commutes with the $\mathbb{Z}_{p}$ charge operator $Q_{p}$ of Eq. (47). It is useful to notice that the projectors $P_{q}=P_{q}^{2}=P_{q}^{\dagger}$ onto the sectors of total $\mathbb{Z}_{p}$ charge $\omega^{q}$ can be computed as

$$
P_{q}=\frac{1}{p} \sum_{m=0}^{p-1} \bar{\omega}^{m q} Q_{p}^{m}
$$

Self-dual representations have two very useful general properties. First, recall the relative prime decomposition of $p$, Eq. (13), and suppose we have self-dual representations $\rho_{\text {sd }}^{\left(r_{j}\right)}$ with coefficients $\alpha_{m}^{\left(r_{j}\right)}, \beta_{m}^{\left(r_{j}\right)}, m=0, \ldots, r_{j}-1$. Then one can exploit the canonical embedding, Eq. (22), to transform the self-dual representations $\rho_{\text {sd }}^{\left(r_{j}\right)}$ of dimensions $r_{j}$ to self-dual representations of dimension $p$,

$$
\rho_{\mathrm{sd}}^{\left(r_{j}\right)} \hookrightarrow \rho_{\mathrm{sd}, j}^{(p)}
$$

with

$$
\begin{aligned}
\rho_{\mathrm{sd}, j}^{(p)}\left(\sigma_{2 i-1}\right) & =\frac{1}{\sqrt{r_{j}}} \sum_{m=0}^{r_{j}-1} \alpha_{m}^{\left(r_{j}\right)}\left(U_{i}^{\dagger}\right)^{m p / r_{j}}, & & i=1, \ldots, M, \\
\rho_{\mathrm{sd}, j}^{(p)}\left(\sigma_{2 i}\right) & =\frac{1}{\sqrt{r_{j}}} \sum_{m=0}^{r_{j}-1} \beta_{m}^{\left(r_{j}\right)}\left(V_{i} V_{i+1}^{\dagger}\right)^{m p^{2} / r_{j}^{2}}, & & i=1, \ldots, M-1 .
\end{aligned}
$$

A key characteristic of these embedded representations is the absence of certain powers of the Weyl generators in the expansion of $\rho_{\mathrm{sd}, j}^{(p)}$, that is, the vanishing of some of the coefficients $\alpha_{m}$ and/or $\beta_{m}$.

Second, the tensor product of two self-dual representations of dimensions $p_{1}, p_{2}$ is canonically isomorphic to a self-dual representation of dimension $p_{1} p_{2}$, provided $p_{1}, p_{2}$ are relative primes. We can state this property most elegantly in terms of the mapping $\cong$ of Eq. (14),

$$
\rho_{\mathrm{sd}}^{\left(p_{1}\right)} \otimes \rho_{\mathrm{sd}}^{\left(p_{2}\right)} \cong \rho_{\mathrm{sd}}^{\left(p_{1} p_{2}\right)}
$$


This canonical identification preserves a very important property. Suppose neither $\rho_{\mathrm{sd}}^{\left(p_{1}\right)}$ nor $\rho_{\mathrm{sd}}^{\left(p_{2}\right)}$ contain vanishing coefficients. Then $\rho_{\mathrm{sd}}^{\left(p_{1} p_{2}\right)}$ on the righthand side of Eq. (73) contains no vanishing coefficients either.

The next crucial task is to actually find self-dual representations, that is, solutions of Eqs. (61) and (62). We will focus on solutions without vanishing coefficients, since we know that solutions with vanishing coefficients have an interpretation in terms of embedded representations.

\section{1. $p$ odd}

For $p=3,5, \ldots$ odd it is convenient to impose the extra constraint

$$
\alpha_{m}=\beta_{m}, \quad m=0, \ldots, p-1 .
$$

Then there exist $2 p$ solutions with no vanishing coefficients. However, it suffices to consider only the conjugate pair of solutions

$$
\alpha_{m}=\beta_{m}=\left\{\begin{array}{l}
\omega^{m(m-1) / 2} \\
\bar{\omega}^{m(m-1) / 2}
\end{array} .\right.
$$

Any other one of the remaining $2(p-1)$ determines a self-dual representation equivalent to one of these two. Furthermore, if $p$ is a prime of the form $p=4 n+1$, the two solutions of Eq. (75) determine unitarily equivalent representations.

The special role of $p=4 n+1$ prime can be understood as follows. The sequence of odd primes can be split into two subsequences of the form $4 n+1$ $(5,13,17, \ldots)$ and $4 n+3(3,7,11, \ldots)$. Also, for $p$ prime (and only for $p$ prime), the set of remainders modulo $p, \mathbb{Z}_{p}=0,1, \ldots, p-1$, becomes a discrete field under modular addition and multiplication. But the polynomial equation $x^{2}+1 \equiv 0$ (modulo $p$ ) has a solution in $\mathbb{Z}_{p}$ only if $p$ belongs to the $4 n+1$ sequence.

Note that the Gaussian representation of the braid group described in Ref. [19] can be reinterpreted as a self-dual representation with $\alpha_{m} \neq \beta_{m}$. The Gaussian representation $[19,20]$ is defined for any $p$ odd as

$$
\rho_{\mathrm{G}}^{(p)}\left(\sigma_{k}\right)=\frac{1}{\sqrt{p}} \sum_{m=0}^{p-1} \omega^{m^{2}} u_{k}^{m}, \quad k=1, \ldots, L-1,
$$

(we change the normalization relative to Ref. [19]) in terms of $L-1$ generators $u_{k}$ satisfying

$$
\begin{aligned}
u_{k}^{p} & =\mathbb{1}, & k & =1, \ldots, L-1, \\
u_{k} u_{k+1} & =\omega^{2} u_{k+1} u_{k}, & k & =1, \ldots, L-2,
\end{aligned}
$$


and commuting otherwise. There exists a faithful, irreducible representation of these relations in terms of Weyl generators,

$$
u_{k} \mapsto\left\{\begin{array}{lll}
U_{i}^{\dagger 2} & \text { if } k=2 i-1, \quad i=1, \ldots, M \\
V_{i} V_{i+1}^{\dagger} & \text { if } k=2 i, \quad i=1, \ldots, M-1 \\
V_{M} & \text { if } k=2 M &
\end{array}\right.
$$

with $L=2 M+1$ as before. Then the Weyl realization of the Gaussian representation is self-dual,

$$
\begin{array}{rlrl}
\rho_{\mathrm{G}}^{(p)}\left(\sigma_{2 i-1}\right) & =\frac{1}{\sqrt{p}} \sum_{m=0}^{p-1} \alpha_{m} U_{i}^{\dagger m}, & & i=1, \ldots, M, \\
\rho_{\mathrm{G}}^{(p)}\left(\sigma_{2 i}\right) & =\frac{1}{\sqrt{p}} \sum_{m=0}^{p-1} \beta_{m}\left(V_{i} V_{i+1}^{\dagger}\right)^{m}, & i=1, \ldots, M-1, \\
\rho_{\mathrm{G}}^{(p)}\left(\sigma_{2 M}\right) & =\frac{1}{\sqrt{p}} \sum_{m=0}^{p-1} \beta_{m}\left(V_{M}\right)^{m},
\end{array}
$$

with

$$
\beta_{m}=\omega^{m^{2}}=\alpha_{2 m(\bmod p)}, \quad m=0, \ldots, p-1 .
$$

Unlike our previous solution, Eq. (75), the Gaussian solution has $\alpha_{m} \neq \beta_{m}$, but the two sets of coefficients are connected by a permutation. The reason is that, since $p$ is assumed to be odd, 2 and $p$ are relative primes. Then, the mapping

$$
m \mapsto 2 m(\bmod p), \quad m=0, \ldots, p-1,
$$

is one-to-one and onto, that is, a permutation.

The fact that the Gaussian representation is self-dual could potentially have practical implications, since there exist recent proposals to realize the Gaussian representation of $B_{2 M}$ in mesoscopic arrays [4, 27].

\section{2. $p=2^{n}, n=1,2, \ldots$}

While we have reasons to believe that self-dual representations with $p=$ $2^{n}$ follow a pattern analogous to the one found for $p$ odd, it remains an open problem to find the non-trivial solutions of Eqs. (61) and (62) for arbitrary $p=2^{n}$. For small $n$ it is possible to solve the equations by elementary algebra. Again we impose the extra constraint Eq. (74). 
For $n=1$, that is, $p=2$, we find only two solutions

\begin{tabular}{|c|c|}
\hline$\alpha_{0}$ & $\alpha_{1}$ \\
\hline \hline 1 & $\mathrm{i}$ \\
\hline 1 & $-\mathrm{i}$ \\
\hline
\end{tabular}

They define unitarily equivalent self-dual representations. Recalling the connection between Majorana fermions and parafermions for $p=2$, we obtain that our self-dual representation reduces to the Majorana representation

$$
\rho_{\text {sd }}^{(2)}\left(\sigma_{2 i-1}\right)=\frac{\mathbb{1} \mp b_{i} a_{i}}{\sqrt{2}}, \quad \rho_{\text {sd }}^{(2)}\left(\sigma_{2 i}\right)=\frac{\mathbb{1} \mp a_{i+1} b_{i}}{\sqrt{2}}
$$

first described in Ref. [13]. (The two signs correspond to the two unitarily equivalent representations of the table above.) This representation of the braid group amounts to a Fock representation, since it can be rewritten in terms of creation and annihilation operators for ordinary fermions,

$$
\begin{aligned}
\rho_{\mathrm{sd}}^{(2)}\left(\sigma_{2 i-1}\right) & =\frac{\mathbb{1} \mp \mathrm{i}\left(2 C_{i}^{\dagger} C_{i}-\mathbb{1}\right)}{\sqrt{2}} \\
\rho_{\mathrm{sd}}^{(2)}\left(\sigma_{2 i}\right) & =\frac{\mathbb{1} \pm \mathrm{i}\left(C_{i}^{\dagger} C_{i+1}+C_{i+1}^{\dagger} C_{i}+C_{i+1} C_{i}+C_{i}^{\dagger} C_{i+1}^{\dagger}\right)}{\sqrt{2}} .
\end{aligned}
$$

Notice the emergence of the anomalous pairing term $C_{i} C_{i+1}+C_{i}^{\dagger} C_{i+1}^{\dagger}$.

Let us illustrate in passing the notion of embedded representation. Suppose for example that $p=2 q$, with $q$ odd. Then from Eqs. (71) and (85) we obtain the $p$ dimensional representations

$$
\begin{aligned}
\rho_{\mathrm{sd}}^{(2 q)}\left(\sigma_{2 i-1}\right) & =\frac{\mathbb{1} \pm \mathrm{i} U_{i}^{\dagger q}}{\sqrt{2}}=\frac{\mathbb{1} \pm \mathrm{i}\left(\Delta_{i}^{\dagger} \Gamma_{i}\right)^{q}}{\sqrt{2}}, \\
\rho_{\mathrm{sd}}^{(2 q)}\left(\sigma_{2 i}\right) & =\frac{\mathbb{1} \pm \mathrm{i}\left(V_{i} V_{i+1}^{\dagger}\right)^{q}}{\sqrt{2}}=\frac{\mathbb{1} \pm \mathrm{i}\left(\Delta_{i} \Gamma_{i+1}^{\dagger}\right)^{q}}{\sqrt{2}},
\end{aligned}
$$

with only two non-vanishing coefficients.

For $n=2$, that is, $p=4$, we find two classes of solutions, each class containing four possible sets of coefficients,

\begin{tabular}{|c|c|c|c|}
\hline$\alpha_{0}$ & $\alpha_{1}$ & $\alpha_{2}$ & $\alpha_{3}$ \\
\hline \hline 1 & $\mathrm{i}$ & -1 & $\mathrm{i}$ \\
\hline 1 & $-\mathrm{i}$ & -1 & $-\mathrm{i}$ \\
\hline 1 & -1 & 1 & 1 \\
\hline 1 & 1 & 1 & -1 \\
\hline
\end{tabular}

\begin{tabular}{|c|c|c|c|}
\hline$\alpha_{0}$ & $\alpha_{1}$ & $\alpha_{2}$ & $\alpha_{3}$ \\
\hline \hline 1 & $\mathrm{i}$ & 1 & $-\mathrm{i}$ \\
\hline 1 & $-\mathrm{i}$ & 1 & $\mathrm{i}$ \\
\hline 1 & 1 & -1 & 1 \\
\hline 1 & -1 & -1 & -1 \\
\hline
\end{tabular}


The representations in a given class are unitarily equivalent. Moreover, the representation associated to the left class of solutions are unitarily equivalent the product representation $\rho_{\text {sd }}^{(2)} \otimes \rho_{\text {sd }}^{(2)}$.

The conclusion of the previous paragraph suggests an interesting conjecture. It may be that for arbitrary $n, p=2^{n}$, there exist $n$ classes of solutions of Eqs. (61) and (62), with one class at least unitarily equivalent to the product representation

$$
\rho_{\text {sd }}^{(2)} \otimes \cdots \otimes \rho_{\text {sd }}^{(2)} \quad(n \text { factors })
$$

If this is true, it poses a potentially non-trivial constraint on that class of representations $\rho_{\mathrm{sd}}^{\left(2^{n}\right)}$. The reason is that the representation $\rho_{\mathrm{sd}}^{(2)}$ has finite period 8,

$$
\left(\rho_{\text {sd }}^{(2)}\left(\sigma_{k}\right)\right)^{8}=\rho_{\text {sd }}^{(2)}\left(\sigma_{k}^{8}\right)=\mathbb{1}, \quad \forall k
$$

It follows that the product representation (91) has period 4 for $n$ even, or period 8 for $n$ odd. If one exists, the same must hold for an equivalent self-dual representation $\rho_{\text {sd }}^{\left(2^{n}\right)}$.

\subsection{General $p$}

It will be easy to construct interesting solutions for arbitrary $p$ once the special case $p=2^{n}$ is completely solved. The reason is that an an arbitrary $p$ can always be decomposed as $p=2^{n} q$, with $q$ odd. This is fortunate, because $2^{n}$ and $q$ are by definition relative primes, and so it is possible to use Eq. (73) to obtain a self-dual representation (with no vanishing coefficients!) for any $\mathrm{p}$,

$$
\rho_{\text {sd }}^{\left(2^{n}\right)} \otimes \rho_{\text {sd }}^{(q)} \cong \rho_{\text {sd }}^{\left(2^{n} q\right)}
$$

The resulting representation on the right-hand side will not in general satisfy the extra condition Eq. (74), even if $\rho_{\mathrm{sd}}^{\left(2^{n}\right)}$ and $\rho_{\mathrm{sd}}^{(q)}$ do satisfy it individually. Specially interesting are the representations obtained for $p=4 q$ combining our solutions by this procedure.

\section{Fock parafermions}

The Majorana representation of the braid group Eq. (86) is one of the most studied in physics $[13,11]$. We think this is in part because it has a Fock space interpretation in terms of ordinary fermions, Eq. (87). The key point is that this interpretation immediately suggests the right quantum 
state of matter to naturally support this type of braiding: electron vacua with anomalous pairing. These observations are typically summarized in the literature as conditions for the emergence of Majorana fermions in condensed matter systems $[37,11]$.

Parafermions with $p \geq 3$ offer a natural generalization of the Majorana braiding paradigm. As we established in the previous Section, there exist a variety of representations of the braid group naturally linked to parafermions through Eq. (68). There also exist proposals to realize parafermions as zeroenergy modes [29] in mesoscopic arrays [28, 4, 49]. Hence the following question becomes relevant: Do particles with exotic $p$-exclusion, $\theta=2 \pi / p$ exchange statistics, and a well defined associated Fock space exist? In this section we will answer this question in the affirmative by introducing Fock spaces $\mathcal{F}_{p}(M)$ of indistinguishable particles satisfying the required statistical conditions ( $M$ is the number of available orbitals). We call these particles Fock parafermions. For $p=2$, Fock parafermions are just ordinary fermions, but for $p>2$ they are anyons with unconventional exclusion and exchange statistics. One of our goals is to obtain an expansion of parafermions in terms of creation and annihilation operators of Fock parafermions.

\subsection{The Fock space of Fock parafermions}

In this paper we propose to construct the Fock space of of indistinguishable, independent particles in terms of two fundamental pieces of information:

1. the state space of a single particle $\mathcal{H}$ (the available orbitals), and

2. a rule to multiply $N$ single-particle states to generate an $N$-body state with the correct exchange and exclusion statistics.

Hence a Fock space is a state space (a Hilbert space defined over the field of the complex numbers $\mathrm{C}$ ) endowed with a physically motivated, associative multiplication. Once a Fock space is specified in this fashion, the unintuitive in general algebra of creation and annihilation operators can be derived systematically. In particular, we will see that the algebra of creation and annihilation operators for Fock parafermions displays a variety of unconventional features that would have been extremely hard to guess a priori.

Let us start by specifying the single-particle state space $\mathcal{H}$ by choosing a basis of orthonormal orbitals $\phi_{1}, \ldots, \phi_{M}$, and suppose for now that the Fock parafermions are independent particles (we can always add interactions later). Then we can specify a many-body state by stating that there are $n_{1}$ Fock parafermions occuppying orbital $\phi_{1}, n_{2}$ Fock parafermions occuppying orbital 
$\phi_{2}$ and so on. Mathematically, we can organize the occupation numbers into a unique object, the ordered list $\left(n_{1}, \ldots, n_{M}\right)$, and assign to this list a unique (up to a phase that we ignore in the following) many-body state,

$$
\left(n_{1}, \ldots, n_{M}\right) \mapsto\left|n_{1}, \ldots, n_{M}\right\rangle \quad \text {. }
$$

Then the general structure of the Fock space is

$$
\mathcal{F}_{p}(M)=\bigoplus_{N=0} \text { Linear } \operatorname{Span}\left\{\left|n_{1}, \ldots, n_{M}\right\rangle \mid \sum_{r=1}^{M} n_{r}=N\right\},
$$

with inner product

$$
\left\langle n_{1}, \cdots, n_{M} \mid n_{1}^{\prime}, \cdots, n_{M}^{\prime}\right\rangle=\prod_{i=1}^{M} \delta\left(n_{i}, n_{i}^{\prime}\right)
$$

The inner product is strongly physically motivated. It guarantees that states with distinct orbital occupations are orthogonal.

This minimal description of the Fock space of Fock parafermions contains no statistical information. First, the range

$$
n_{i}=0,1, \ldots, n_{\mathrm{E}}, \quad i=1, \cdots, M,
$$

of the occupation numbers cannot be specified until we determine the exclusion statistics of Fock parafermions. We call the not necessarily finite integer $n_{\mathrm{E}} \geq 2$ the exclusion parameter. Second, the state of a system of independent Fock parafermions is uniquely specified (up to a phase) by the mapping Eq. (94). In this formalism, the notion of exchanging two Fock parafermions has no mathematical representation. Thus the present description of $\mathcal{F}_{p}(M)$ contains no information on the exchange statistics of Fock parafermions. In the following we will use the abbreviated notation

$$
\begin{aligned}
\left|n_{i}\right\rangle & =\left|0, \ldots, 0, n_{i}, 0, \ldots, 0\right\rangle \\
\left|n_{i}, n_{j}\right\rangle & =\left|0, \ldots, 0, n_{i}, 0, \ldots, 0, n_{j}, 0, \ldots, 0\right\rangle
\end{aligned}
$$

and so on.

To account for both aspects of particle statistics, we define an associative multiplication $\times$ in Fock space in two steps. First, the rule

$$
\left(\left|n_{i}=1\right\rangle\right)^{m} \equiv\left|n_{i}=m\right\rangle=\left|n_{i}=1\right\rangle \times \cdots \times\left|n_{i}=1\right\rangle \quad(m \text { times })
$$


allow us to describe algebraically the process of adding Fock parafermions to any fixed orbital. In light of this definition, the mathematical role of the exclusion parameter becomes clear: the exclusion parameter $n_{\mathrm{E}} \geq 2$ is the smallest integer such that

$$
\left(\left|n_{i}=1\right\rangle\right)^{n_{\mathrm{E}}}=\left|n_{i}=n_{\mathrm{E}}\right\rangle=0 .
$$

Second, the requirement of consistency with the exchange rules for parafermions, Eqs. (28) and (29), suggests the definition

$$
\left|n_{i}\right\rangle \times\left|n_{j}\right\rangle=\omega^{n_{i} n_{j}}\left|n_{j}\right\rangle \times\left|n_{i}\right\rangle \equiv\left|n_{i}, n_{j}\right\rangle \quad \text { for } i<j
$$

( $\left.\omega=e^{\mathrm{i} \theta}, \theta=2 \pi / p\right)$ for combining two different orbitals with arbitrary occupation. This multiplication rule captures the exchange statistics of Fock parafermions.

With these definitions, we see that any state in Fock space can be generated by multiplying single-particle states as follows

$$
\left|n_{1}, \ldots, n_{M}\right\rangle=(|1,0, \ldots, 0\rangle)^{n_{1}} \times \cdots \times(|0, \ldots, 0,1\rangle)^{n_{M}} .
$$

Hence we see that the Fock vacuum

$$
|0\rangle \equiv\left|n_{1}=0, \ldots, n_{M}=0\right\rangle=(|1,0, \ldots, 0\rangle)^{0} \times \cdots \times(|0, \ldots, 0,1\rangle)^{0}
$$

plays the distinguished role of multiplicative identity,

$$
\left|n_{1}, \ldots, n_{M}\right\rangle \times|0\rangle=|0\rangle \times\left|n_{1}, \ldots, n_{M}\right\rangle=\left|n_{1}, \ldots, n_{M}\right\rangle .
$$

Finally, we need to specify the exclusion parameter $n_{\mathrm{E}}$ for Fock parafermions. Remarkably, $n_{\mathrm{E}}$ is determined by the exchange angle $\theta$, the basic physical considerations leading to the inner product Eq. (96), and the following assumption: any state $|\Psi\rangle$ in Fock space that commutes with every other state $|\Phi\rangle \in \mathcal{F}_{p}(M)$ is a scalar multiple of the Fock vacuum,

$$
|\Psi\rangle \times|\Phi\rangle=|\Phi\rangle \times|\Psi\rangle, \quad \forall|\Phi\rangle \quad \Rightarrow \quad|\Psi\rangle=\alpha|0\rangle, \quad \alpha \in \mathbb{C} .
$$

We can understand this assumption as the algebraic statement reflecting the uniqueness of the Fock vacuum. Later we will see that this assumption implies any operator in Fock space that commutes with every parafermionic creation (or annihilation) operator is a scalar multiple of the identity operator. 
The determination of $n_{\mathrm{E}}$ starts with the observation that

$$
\left|n_{i}=p\right\rangle \times\left|n_{j}\right\rangle=\left|n_{j}\right\rangle \times\left|n_{i}=p\right\rangle,
$$

thanks to Eqs. (97), (99), and $\omega^{p}=1$, and so the $M$ states $\left|n_{i}=p\right\rangle$ satisfy

$$
\left|n_{i}=p\right\rangle=\alpha_{i}|0\rangle, \quad i=1, \cdots, M,
$$

according to our assumption, Eq. (103). But since these states contain $p$ particles,

$$
0=\left\langle 0 \mid n_{i}=p\right\rangle=\alpha_{i} .
$$

It follows that

$$
\left|n_{i}=p\right\rangle=\left(\left|n_{i}=1\right\rangle\right)^{p}=0 .
$$

We conclude that $n_{\mathrm{E}}=p$, i.e., Fock parafermions satisfy $p$-exclusion and $2 \pi / p$-exclusion. Thus, the dimension of the Fock space of Fock parafermions is

$$
\operatorname{dim}_{\mathrm{C}} \mathcal{F}_{p}(M)=p^{M} .
$$

In the mathematical literature, the algebra $\mathcal{F}_{p}(M)$ is known as the $p$ Grassmann algebra with $M$ generators [36, 34]. Its many-body interpretation seems to have gone unnoticed up to now, for reasons discussed in Section 6. Let us notice in closing that $\mathcal{F}_{2}(M)$ is just the standard Fock space of indistinguishable fermions with $M$ available orbitals $[40,50]$. This will become self-evident in the next section when we compute the algebra of creation and annihilation operators associated to $\mathcal{F}_{p}(M)$.

\subsection{Creation and annihilation operators}

By definition, a single application of a creation operator for orbital $i$ adds a particle in that orbital to any many-body state. Our proposal is to define creation operators $C_{i}^{\dagger}$ of Fock parafermions in terms of the multiplication of Fock states introduced above. So let

$$
\begin{aligned}
C_{i}^{\dagger}\left|n_{1}, \ldots, n_{M}\right\rangle & \equiv\left|n_{i}=1\right\rangle \times\left|n_{1}, \ldots, n_{M}\right\rangle \\
& =\bar{\omega}^{\sum_{j<i} n_{j}}\left|n_{1}, \ldots, n_{i}+1, \ldots, n_{M}\right\rangle .
\end{aligned}
$$

Because we know the inner product from Eq. (96), we can immediately compute the adjoint annihilation operators,

$$
C_{i}\left|n_{1}, \ldots, n_{M}\right\rangle=\omega^{\sum_{j<i} n_{j}}\left|n_{1}, \ldots, n_{i}-1, \ldots, n_{M}\right\rangle .
$$


We can also define number operators as

$$
N_{i}\left|n_{1}, \ldots, n_{i}, \ldots, n_{M}\right\rangle=n_{i}\left|n_{1}, \ldots, n_{i}, \ldots, n_{M}\right\rangle .
$$

The creation operators satisfy

$$
C_{i}^{\dagger p}=0, \quad C_{i}^{\dagger} C_{j}^{\dagger}=\omega C_{j}^{\dagger} C_{i}^{\dagger} \quad(i<j),
$$

and analogous relations follow for the annihilation operators,

$$
C_{i}^{p}=0, \quad C_{i} C_{j}=\omega C_{j} C_{i} \quad(i<j) .
$$

Creation and annihilation operators for different orbitals commute up to a phase,

$$
C_{i}^{\dagger} C_{j}=\bar{\omega} C_{j} C_{i}^{\dagger}, \quad C_{i} C_{j}^{\dagger}=\bar{\omega} C_{j}^{\dagger} C_{i} \quad(i<j) .
$$

But what are the relations for $C \mathrm{~s}$ and $C^{\dagger} \mathrm{s}$ for a given orbital $i$ ? Canonical fermions require one relation per orbital, Eq. (35). In contrast, Fock parafermions require $p-1$ relations,

$$
C_{i}^{\dagger m} C_{i}^{m}+C_{i}^{p-m} C_{i}^{\dagger(p-m)}=\mathbb{1} \quad m=1, \cdots, p-1 .
$$

This section's relations summarize the algebra of creation and annihilation operators of Fock parafermions. For $p=2$, they reduce to the standard fermionic algebra.

One of the landmark features of canonical fermions and bosons, and also some algebraic frameworks for Abelian anyons [16], is the simple relation between the creation and annihilation operators and number operators. This relation is somewhat less simple for Fock parafermions since

$$
N_{i}=\sum_{m=1}^{p-1} C_{i}^{\dagger m} C_{i}^{m}
$$

However, it is still true that $N_{i}$ is the generator of $U(1)$ transformations. To see this, notice that

$$
\left[C_{i}^{\dagger m} C_{i}^{m}, C_{i}\right]=C_{i}^{\dagger m} C_{i}^{m+1}-C_{i}^{\dagger m-1} C_{i}^{m}
$$

from Eq. (115). It follows that

$$
\left[N_{i}, C_{i}^{\dagger}\right]=C_{i}^{\dagger}, \quad\left[N_{i}, C_{i}\right]=-C_{i} .
$$


Any operator in Fock space has a normal form : $\mathcal{O}$ :, defined implicitly as

$$
\mathcal{O}=: \mathcal{O}:+\langle 0|\mathcal{O}| 0\rangle, \quad: \mathcal{O}:|0\rangle=0=: \mathcal{O}:^{\dagger}|0\rangle .
$$

Remarkably, the unconventional algebra of creation and annihilation operators for Fock parafermions provides a systematic way to compute : $\mathcal{O}$ : by repeated application of Eqs. (112), (113), (114), and (115) to put all the creation operators to the left of every annihilation operator. But for fermions $(p=2)$ a more efficient procedure exists: the algorithm known as Wick's theorem [50]. We do not know whether some generalization of Wick's theorem exists that applies to Fock parafermions.

Let us note in closing that if one takes the view that the orbitals denote sites on a latttice (localized orbitals), Fock parafermions can be defined in any number of dimensions. This point is directly linked to the analogous discussion for parafermions around Eq. (36), and we will clarify it further in Appendix A.

\section{Fock representation of Weyl parafermions}

In this section we exploit the Fock parafermions just defined to construct a Fock space representation of parafermions. This construction will generalize the representation of Majoranas in terms of ordinary fermions to all $p$.

Unfortunately, Eq. (34) as it stands suggests the wrong starting point. Let $\kappa \equiv e^{\mathrm{i} \pi / p}$, so that $\kappa^{2}=\omega$. Then the combination

$$
\chi_{i} \equiv \Gamma_{i}+\kappa \Delta_{i},
$$

satisfies $[34,36]$

$$
\chi_{i}^{p}=0, \quad \chi_{i} \chi_{j}=\omega \chi_{j} \chi_{i} \quad(i<j) .
$$

Moreover, for $p=2, \chi_{i}=2 C_{i}$ and $\chi_{i}^{\dagger}=2 C_{i}^{\dagger}$ are proportional to fermionic annihilation and creation operators. This suggest that we set $C_{i} \propto \chi_{i}, C_{i}^{\dagger} \propto \chi_{i}^{\dagger}$, for all $p$. But this ansatz fails to satisfy Eq. (115). This, in our opinion, seems to be the reason why the algebra of creation and annihilation operators described in this paper is new. The natural candidates for creation and annihilation operators associated to parafermions, the $\chi_{i}$ of Eq. (120) long known in the literature [34, 36], do not satisfy the correct algebra (in particular, they cannot be normal-ordered) to grant a particle interpretation. 
The correct starting point can be obtained from rewriting Eq. (34) in the form

$$
\Gamma_{i}=a_{i}=C_{i}+C_{i}^{\dagger}, \quad \Delta_{i}=-\mathrm{i} b_{i}=\left(C_{i}+C_{i}^{\dagger}\right)(-1)^{C_{i}^{\dagger} C_{i}},
$$

which suggests a suitable generalization,

$$
\Gamma_{i} \equiv C_{i}+C_{i}^{\dagger p-1}, \quad \Delta_{i} \equiv\left(C_{i}+C_{i}^{\dagger p-1}\right) \omega^{N_{i}}
$$

valid for all $p$. This is the Fock representation of parafermions we have been looking for, and a main result of this paper.

The number operator $N_{i}$ can be eliminated from Eq. (123) with the use of the identity

$$
\omega^{N_{i}}=\mathbb{1}+(\omega-1) \sum_{m=1}^{p-1} \omega^{m-1} C_{i}^{\dagger m} C_{i}^{m}
$$

that reduces to the well known $(-1)^{C_{i}^{\dagger} C_{i}}=1-2 C_{i}^{\dagger} C_{i}$ for ordinary fermions $(p=2)$. Since, by Eq. (115),

$$
C_{i} C_{i}^{\dagger m} C_{i}^{m}=C_{i}^{\dagger m-1} C_{i}^{m}
$$

we have that

$$
\Gamma_{i}=C_{i}+C_{i}^{\dagger p-1}, \quad \Delta_{i}=\omega C_{i}+C_{i}^{\dagger p-1}+(\omega-1) \sum_{m=2}^{p-1} C_{i}^{\dagger m-1} C_{i}^{m}
$$

It is possible to invert Eq. (123) to obtain an expansion of creation and annihilation operators for Fock parafermions in terms of parafermions. This expansion follows most easily from results obtained in the Appendix A, so here we just quote the expressions,

$$
\begin{aligned}
C_{i} & =\frac{p-1}{p} \Gamma_{i}-\frac{1}{p} \sum_{m=1}^{p-1} \omega^{m(m+1) / 2} \Gamma_{i}^{m+1} \Delta_{i}^{\dagger m}, \\
C_{i}^{\dagger} & =\frac{p-1}{p} \Gamma_{i}^{\dagger}-\frac{1}{p} \sum_{m=1}^{p-1} \bar{\omega}^{m(m+1) / 2} \Delta_{i}^{m}\left(\Gamma_{i}^{\dagger}\right)^{m+1} .
\end{aligned}
$$


Finally, let us exploit Eqs. (53) and (123) to reinterpret the vector Potts model as the Hamiltonian for a system of Fock parafermions $\left(J_{M}=0\right)$,

$$
\begin{array}{r}
H_{\mathrm{VP}}=-\frac{1}{2}\left[\sum_{i=1}^{M} h_{i} \bar{\omega}^{N_{i}}+\sum_{i=1}^{M-1} J_{i}\left(C_{i} C_{i+1}^{\dagger}+C_{i}^{\dagger p-1} C_{i+1}^{p-1}\right) \omega^{N_{i}}\right. \\
\left.+\sum_{i=1}^{M-1} J_{i}\left(C_{i} C_{i+1}^{p-1}+C_{i}^{\dagger p-1} C_{i+1}^{\dagger}\right) \omega^{N_{i}}\right]+ \text { h.c. }
\end{array}
$$

From this point of view, the vector Potts Hamiltonian splits into two terms, Eqs. (128) and (129), with different symmetry properties. The first term, Eq. (128), is $U(1)$ symmetric because it commutes with the operator $\hat{N}=$ $\sum_{i=1}^{M} N_{i}$ counting the total number of Fock parafermions. The second type of term, Eq. (129), breaks this continous symmetry down to a discrete $\mathbb{Z}_{p}$ symmetry $\omega^{\hat{N}}$ via anomalous pairing. The existence of an emergent $U(1)$ symmetry in the vector Potts model and its exact nature was a subject of discussion for many years, see Ref. [2] and references therein. From the present analysis we can understand these features in terms of particle conservation broken down, due to anomalous pairing, to a discrete symmetry.

\section{Acknowledgements}

EC dedicates this paper to the memory of China Nieves Corriente de and Alberto Glavocich, and gratefully acknowledges support from the Dutch Science Foundation NWO/FOM and an ERC Advanced Investigator Grant.

\section{Appendix A. Weyl hard-core bosons}

In the main body of the paper we established "dictionaries" [16] connecting Weyl generators to parafermions, Eqs. (26) and (27), and Fock parafermions to parafermions, Eqs. (123) and (127). In this appendix we would like to combine some of these results to obtain a Fock representation of Weyl generators that helps in the derivation of Eq. (127). A simple calculation reveals a new Fock space of particles satisfying bosonic exchange statistics and $p$ exclusion. We call these particles Weyl hard-core bosons. The latter provide a local Fock space description of Weyl generators, just like Fock parafermions are best suited for the local Fock space description of parafermions. The creation and annihilation operators for Weyl hard-core bosons are connected to those of Fock parafermions by a Jordan-Wigner-like transformation. 
Consider expressing the Weyl generators in terms of parafermions. From Eqs. (27), and (123)

$$
U_{i}=\Gamma_{i}^{\dagger} \Delta_{i}=\left(C_{i}^{\dagger}+C_{i}^{p-1}\right)\left(C_{i}+C_{i}^{\dagger p-1}\right) \omega^{N_{i}}=\omega^{N_{i}},
$$

which follows from p-exclusion and Eq. (115), and $\left(\left(\omega^{N_{i}}\right)^{\dagger}=\bar{\omega}^{N_{i}}\right)$

$$
\begin{aligned}
V_{i} & =\Gamma_{i}\left(\prod_{j=1}^{i-1} \Delta_{j}^{\dagger} \Gamma_{j}\right)=\left(C_{i}+C_{i}^{\dagger p-1}\right)\left(\prod_{j=1}^{i-1} \bar{\omega}^{N_{j}}\right)=C_{i} \prod_{j=1}^{i-1} \bar{\omega}^{N_{j}}+\left(C_{i}^{\dagger} \prod_{j=1}^{i-1} \omega^{N_{j}}\right)^{p-1} \\
& =B_{i}+B_{i}^{\dagger p-1}
\end{aligned}
$$

where we have defined creation and annihilation operators

$$
B_{i}^{\dagger}=C_{i}^{\dagger} \prod_{j=1}^{i-1} \omega^{N_{j}}, \quad B_{i}=C_{i} \prod_{j=1}^{i-1} \bar{\omega}^{N_{j}}
$$

Noticing that

$$
N_{i}=\sum_{m=1}^{p-1} C_{i}^{\dagger m} C_{i}^{m}=\sum_{m=1}^{p-1} B_{i}^{\dagger m} B_{i}^{m}
$$

we thus managed to express the Weyl generators in terms of $B_{i}$, and $B_{i}^{\dagger}$.

The key point is that these operators satisfy bosonic-type commutation relations

$$
\left[B_{i}, B_{j}\right]=0, \quad\left[B_{i}, B_{j}^{\dagger}\right]=0, \quad\left[B_{i}^{\dagger}, B_{j}^{\dagger}\right]=0 .
$$

Furthermore,

$$
B_{i}^{p}=0=B_{i}^{\dagger p}, \quad B_{i}^{\dagger m} B_{i}^{m}+B_{i}^{p-m} B_{i}^{\dagger(p-m)}=\mathbb{1} \quad(m=1, \cdots, p-1),
$$

with number operators, $N_{i}=\sum_{m=1}^{p-1} B_{i}^{\dagger m} B_{i}^{m}$, which are generators of $U(1)$ transformations,

$$
\left[N_{i}, B_{i}\right]=-B_{i}, \quad\left[N_{i}, B_{i}^{\dagger}\right]=B_{i}^{\dagger}
$$

These creation and annihilation operators are the Weyl hard-core bosons alluded to at the beginning of this appendix. Just as for Fock parafermions, any operator polynomial in the $B_{i}^{\dagger}, B_{i}$ can be put in normal form just by repeated application of the defining relations (A.5) and (A.6). 
Equations (A.5) and (A.6) imply that the Fock space of Weyl hard-core bosons describes bosonic $\theta=0$ exchange and $p$-exclusion statistics,

$$
\left|n_{i}\right\rangle \times\left|n_{j}\right\rangle=\left|n_{j}\right\rangle \times\left|n_{i}\right\rangle=\left|n_{i}, n_{j}\right\rangle, \quad\left(\left|n_{i}=1\right\rangle\right)^{p}=0 .
$$

Notice that in this case the exclusion and exchange statistics are independent. This is clear since the latter depends on $p$ while the former is fixed.

Weyl hard-core bosons can be expanded directly in terms of Weyl generators. Since

$$
\left\langle n_{i}=m\left|V_{i}^{\dagger} B_{i}\right| n_{i}=n\right\rangle=\left\{\begin{array}{ll}
0 & \text { if } \quad n \neq m \\
0 & \text { if } \quad n=m=0 \\
1 & \text { if } \quad n=m=1, \ldots, p-1
\end{array},\right.
$$

the diagonal operator $V_{i}^{\dagger} B_{i}$ can be expanded as $V_{i}^{\dagger} B_{i}=\sum_{m=0}^{p-1} c_{m} U_{i}^{\dagger m}$, with expansion coefficients $c_{m}=\operatorname{tr}\left(U_{i}^{m} V_{i}^{\dagger} B_{i}\right) / p$. It follows that

$$
B_{i}=\frac{p-1}{p} V_{i}-\frac{1}{p} \sum_{m=1}^{p-1} V_{i} U_{i}^{\dagger m}, \quad B_{i}^{\dagger}=\frac{p-1}{p} V_{i}^{\dagger}-\frac{1}{p} \sum_{m=1}^{p-1} U_{i}^{m} V_{i}^{\dagger},
$$

or in matrix representation, as follows from Eq. (7),

$$
B=\left(\begin{array}{ccccc}
0 & 1 & 0 & \cdots & 0 \\
0 & 0 & 1 & \cdots & 0 \\
0 & 0 & 0 & \cdots & 0 \\
\vdots & \vdots & \vdots & & \vdots \\
0 & 0 & 0 & \cdots & 1 \\
0 & 0 & 0 & \cdots & 0
\end{array}\right), \quad B^{\dagger}=\left(\begin{array}{cccccc}
0 & 0 & 0 & \cdots & 0 & 0 \\
1 & 0 & 0 & \cdots & 0 & 0 \\
0 & 1 & 0 & \cdots & 0 & 0 \\
\vdots & \vdots & \vdots & & \vdots & \vdots \\
0 & 0 & 0 & \cdots & 1 & 0
\end{array}\right)
$$

Notice that

$$
B_{i} U_{i}=\omega U_{i} B_{i}, \quad U_{i} B_{i}^{\dagger}=\omega B_{i}^{\dagger} U_{i} .
$$

We have now the elements to derive Eq. (127) of Section 6. By combining Eqs. (A.10) and (A.3), and using the definition of parafermions Eq. (36), we obtain Eq. (127). Fock parafermions can be defined in any number of space dimensions by exploiting the generalized Jordan-Wigner transformation

$$
C_{r}^{\dagger}=B_{r}^{\dagger} \prod_{x<r} U_{x}^{\dagger}, \quad C_{r}=B_{r} \prod_{x<r} U_{x}
$$


with

$$
U_{\boldsymbol{x}}=\omega^{N_{\boldsymbol{x}}}=\mathbb{1}+(\omega-1) \sum_{m=1}^{p-1} \omega^{m-1} B_{\boldsymbol{x}}^{\dagger m} B_{\boldsymbol{x}}^{m} .
$$

Weyl hard-core bosons become the standard hard-core bosons [16] $\left(U_{\boldsymbol{x}}=\right.$ $\sigma_{\boldsymbol{x}}^{z}=1-2 N_{\boldsymbol{x}}$ ) for $p=2$, and Eq. (A.13) reduces to the Matsubara-Matsuda transformation in any number of dimensions.

We finally describe the mathematical connection between Weyl hard-core bosons and an alternative description of hard-core bosons, the so-called $g$-ons introduced in Ref. [16], and later on used in connection to the simulation of competition and coexistence of magnetism and superfluid behaviors in cold atoms and optical lattice systems [51]. Define

$$
g_{i} \equiv B_{i} N_{i}^{1 / 2}, \quad g_{i}^{\dagger} \equiv N_{i}^{1 / 2} B_{i}^{\dagger},
$$

and let $W_{i}=W_{i}^{2}=W_{i}^{\dagger}$ denote the orthogonal projector onto the subspace of states with maximal occupation $p-1$ of orbital $i$. The operators $g_{i}, g_{i}^{\dagger}, W_{i}(i=$ $1, \ldots, M)$ offer an alternative, yet not a Lie algebra, description of hard-core bosons with $p$ exclusion that converges naturally to canonical bosons in the limit $p \rightarrow \infty$, and to standard hard-core bosons for $p=2$. Their algebra is completely specified by the relations

$$
\left[g_{i}, g_{j}\right]=0=\left[g_{i}^{\dagger}, g_{j}^{\dagger}\right], \quad\left[g_{i}, g_{j}^{\dagger}\right]=\delta_{i, j}\left(\mathbb{1}-p W_{i}\right), \quad g_{i}^{\dagger} W_{i}=0=W_{i} g_{i}
$$

and $p$-exclusion [16]. For any $p, N_{i}=g_{i}^{\dagger} g_{i}$. One may argue that if the weak limit

$$
w-\lim _{p \rightarrow \infty} W_{i}=0
$$

holds, then $g_{i}, g_{i}^{\dagger}$ converge to canonical bosons in the limit $p \rightarrow \infty$. A weak limit is established through the convergence of matrix elements. The matrix elements of $W_{i}$ converge to zero because, by construction, there are no physical states with infinite occupation number in Fock space.

Let us emphasize that $g$-ons with $p$-exclusion are best suited for the local Fock space description of spins of dimension $2 S+1=p$. In contrast, an attempt to describe Weyl generators in terms of $g$-ons would return hopelesly complicated expressions (especially for relatively large $p$ ). Conversely, an attempt to describe spins in terms of Weyl hard-core bosons would again 
return complicated expressions (especially for relatively large $S$ ).

\section{References}

\section{References}

[1] J. K. Pachos, Introduction to Topological Quantum Computation (Cambridge University Press, Cambridge, 2012).

[2] G. Ortiz, E. Cobanera, and Z. Nussinov, Nuc. Phys. B 854, 780 (2012).

[3] E. Cobanera, G. Ortiz, and Z. Nussinov, Adv. in Phys. 60, 679 (2011).

[4] N. H. Lindner, E. Berg, G. Refael, and A. Stern, Phys. Rev. X 2, 041002 (2012).

[5] C. Kassel and V. Turaev, Braid Groups (Springer Verlag, New York, 2010).

[6] M.G.G. Laidlaw and C. Morette-de Witt, Phys. Rev. D 6 (1971).

[7] J. M. Leinaas and J. Myrheim, Il Nuovo Cimento B 37, 1 (1977).

[8] A. Shapere and Wilczek, Geometric Phases in Physics, (World Scientific, Singapore, 1988).

[9] G. Rigolin, G. Ortiz, and V. H. Ponce, Phys. Rev. A 78, 052508 (2008); G. Rigolin and G. Ortiz, Phys. Rev. Lett. 104, 170406 (2010); Phys. Rev. A 85, 062111 (2012).

[10] Z. Nussinov and G. Ortiz, Ann. Phys. 324, 977 (2009).

[11] C. W. J. Beenakker, Annu. Rev. Con. Mat. Phys. 4, 113 (2013).

[12] T. Hyart, B. van Heck, I. C. Fulga, M. Burrello, A. R. Akhmerov, and C. W. J. Beenakker, arXiv:1303.4379 [quant-ph] (2013).

[13] D. A. Ivanov, Phys. Rev. Lett. 86, 268 (2001).

[14] Let us mention that the Weyl parafermions, the object of study in this paper, are not the Green parafermions of Ref. [15]. See Ref. [16] for a contemporary perspective on Green parafermions. 
[15] O. W. Greenberg and A. M. L. Messiah, Phys. Rev. 138, B1155 (1965).

[16] C.D. Batista and G. Ortiz, Phys. Rev. Lett. 86, 1082 (2001); Adv. in Phys. 53, 1 (2004).

[17] E. Fradkin and L.P. Kadanoff, Nucl. Phys. B 170, 1 (1980).

[18] F. C. Alcaraz and R. Köberle, Phys. Rev. D 24, 1562 (1981).

[19] V. Jones, Comm. Math. Phys. 125, 459 (1989).

[20] D. Goldschmidt and V. Jones, Geometriae Dedicata 31, 165 (1989).

[21] The reason is remarkably simple. It follows from Eq. (1) that $\left[X_{i}^{n}, P_{i}\right]=$ $i \hbar n X_{i}^{n-1}(n=2,3, \ldots)$. But then a representation of Heisenberg's commutation relations in terms of bounded operators would have to satisfy the inequalities

$$
n \hbar \leq 2\left\|X_{i}\right\| \cdot\left\|P_{i}\right\|, \quad n=2,3, \ldots,
$$

which clearly contradicts the assumption that both $X_{i}$ and $P_{i}$ are bounded. In particular, there is no finite-dimensional representation of Heisenberg's relations.

[22] H. Weyl, The Theory of Groups and Quantum Mechanics (Dover Publications, New York, 1950).

[23] J. Schwinger, Quantum Mechanics: Symbolism of Atomic Measurements (Springer Verlag, Berlin, 2001).

[24] D. B. Fairlie, P. Fletcher, and C. K. Zachos, J. Math. Phys. 31, 1088 (1990).

[25] R. Aldrovandi and D. Galetti, J. Math. Phys. 31, 2987 (1990).

[26] J. Wess and B. Zumino, Nucl.Phys. B (Proc. Suppl.) 18, 302 (1990).

[27] M. B. Hastings, C. Nayak, and Z. Wang, Phys. Rev. B 87, 165421 (2013).

[28] D. J. Clarke, J. Alicea, and K. Shtengel, Nat. Commun. 4, 1348 (2013).

[29] P. Fendley, J. Stat. Mech. P11020 (2012).

[30] G. 't Hooft, Nucl. Phys. B 138, 1 (1978). 
[31] D. Horn, M. Weinstein, and S. Yankielowicz, Phys. Rev. D 19, 3715 (1979).

[32] S. S. Bullock and G. K. Brennen, J. Phys. A: Math. Theor. 40, 3481 (2007).

[33] Several choices of Weyl generators, satisfying the generalized Clifford algebra, are possible. Our particular choice, Eq. (26), is dictated by the convenience of writing the string operator in diagonal form. A different standard choice [3] is given by

$$
\tilde{\Gamma}_{i}=U_{i}\left(\prod_{j=1}^{i-1} V_{j}^{\dagger}\right), \quad \tilde{\Delta}_{i}=V_{i}^{\dagger} \tilde{\Gamma}_{i} .
$$

Another $[18,29]$, uses an additional phase factor in $\tilde{\Delta}_{i}$,

$$
\tilde{\Delta}_{i}=-e^{i \pi / p} V_{i}^{\dagger} \tilde{\Gamma}_{i} .
$$

As explained in Ref. [3] and Section 3 a realization of the generalized Clifford algebra emerges naturally from the study of dualities in quantum clock models. From this viewpoint, the phase $-e^{\mathrm{i} \pi / p}$ has no reason to appear, and the freedom in the definition of parafermions is in correspondence with that associated to the specification of self-duality transformations [3].

[34] A. K. Kwasniewski, J. Math. Phys. 26, 2234 (1985).

[35] T. L. Smith, Pacific J. Math. 149, 157 (1991) and 149, 185 (1991).

[36] M. Rausch de Traubenberg, Adv. Appl. Clifford Alg. 4, 131 (1994) [arXiv:hep-th/9404057].

[37] A. Yu. Kitaev, Phys.-Usp. 44, 131 (2001).

[38] Z. Nussinov, G. Ortiz, and E. Cobanera, Phys. Rev. B 86, 085415 (2012).

[39] S. Deng, L. Viola, and G. Ortiz, Phys. Rev. Lett. 108, 036803 (2012).

[40] E. K. U. Gross, E. Runge, and O. Heinonen, Many-Particle Theory (IOP, Bath, 1991). 
[41] The clock Hamiltonian of Eq. (37) is related to the one studied in Refs. $[3,2]$ by a local unitary transformation, the discrete Fourier transform $F$. The discrete Fourier transform $F$ acts as: $F U F^{\dagger}=V^{\dagger}$, and $F V F^{\dagger}=U$.

[42] E. Cobanera, G. Ortiz, and Z. Nussinov, Phys. Rev. Lett. 104, 020402 (2010).

[43] E. Cobanera, G. Ortiz, and Z. Nussinov, Phys. Rev. B 87, 041105 (2013).

[44] L.P. Kadanoff and H. Ceva, Phys. Rev. B 3, 3918 (1971).

[45] H. O. H. Churchill et. al., Phys. Rev. B 87, 241401(R) (2013).

[46] S. Das Sarma, M. Freedman, C. Nayak, S.H. Simon, and A. Stern, Rev. Mod. Phys. 80, 1083 (2008).

[47] The quantum control model of quantum computation assumes the existence of physical systems that can be controlled by modulating the parameters of the system's Hamiltonian. The control possibilities are used to implement specific quantum gates that represent the unitary evolution of the physical system over a time step obtained by specific modulations of the Hamiltonian. In this regard, we can think of the clock Hamiltonian $H_{\mathrm{VP}}$ of Eq. (37) as our system Hamiltonian whose parameters $\left\{h_{i}, J_{i}\right\}$ are controlled. The quantum control and quantum gate viewpoints are effectively equivalent [48].

[48] G. Ortiz, J. E. Gubernatis, E. Knill, and R. Laflamme, Phys. Rev. A 64, 022319 (2001).

[49] M. Burrello, B. van Heck, and E. Cobanera, Phys. Rev. B 87, 195422 (2013) .

[50] L. E. Ballentine, Quantum Mechanics: A Modern Development (World Scientific, Singapore, 1998).

[51] G. Ortiz and C. D. Batista, in Condensed Matter Theories, Vol. 18, edited by M. de Llano, C. Fiolhais, and J. da Providência (Nova Science Publishers, Inc., Hauppauge, 2003), p. 225; arXiv:cond-mat/0403222. 